Conditions and Impact of Star Formation

Editors: R. Simon, M. Röllig

EAS Publications Series, Vol. ?, 2021

\title{
THE DEUTERATION CLOCK FOR MASSIVE STARLESS CORES
}

\author{
Shuo Kong ${ }^{1}$, Jonathan C. Tan ${ }^{2}$, Paola Caselli ${ }^{3}$ and Francesco Fontani ${ }^{4}$
}

\begin{abstract}
To understand massive star formation requires study of its initial conditions. Two massive starless core candidates, C1-N \& C1-S, have been detected in IRDC G028.37+00.07 in $\mathrm{N}_{2} \mathrm{D}^{+}(3-2)$ with $A L M A$. From their line widths, either the cores are subvirial and are thus young structures on the verge of near free-fall collapse, or they are threaded by $\sim 1 \mathrm{mG} B$-fields that help support them in near virial equilibrium and potentially have older ages. We modeled the deuteration rate of $\mathrm{N}_{2} \mathrm{H}^{+}$to constrain collapse rates of the cores. First, to measure their current deuterium fraction, $D_{\text {frac }}^{\mathrm{N}_{2} \mathrm{H}^{+}} \equiv\left[\mathrm{N}_{2} \mathrm{D}^{+}\right] /\left[\mathrm{N}_{2} \mathrm{H}^{+}\right]$, we observed multiple transitions of $\mathrm{N}_{2} \mathrm{H}^{+}$and $\mathrm{N}_{2} \mathrm{D}^{+}$with CARMA, SMA, JCMT, $N R O 45 \mathrm{~m}$ and IRAM $30 \mathrm{~m}$, to complement the $A L M A$ data. For both cores we derived $D_{\text {frac }}^{\mathrm{N}_{2} \mathrm{H}^{+}} \sim 0.3$, several orders of magnitude above the cosmic $[\mathrm{D}] /[\mathrm{H}]$ ratio. We then carried out chemodynamical modeling, exploring how collapse rate relative to free-fall, $\alpha_{\mathrm{ff}}$, affects the level of $D_{\text {frac }}^{\mathrm{N}_{2} \mathrm{H}^{+}}$that is achieved from a given initial condition. To reach the observed $D_{\text {frac }}^{\mathrm{N}_{2} \mathrm{H}^{+}}$, most models require slow collapse with $\alpha_{\mathrm{ff}} \sim 0.1$, i.e., $\sim 1 / 10$ th of free-fall. This makes it more likely that the cores have been able to reach a near virial equilibrium state and we predict that strong $B$-fields will eventually be detected. The methods developed here will be useful for measurement of the pre-stellar core mass function.
\end{abstract}

\section{Deuteration as a Tracer of Massive Starless Core Dynamics}

Massive star formation involves many open questions, in part because examples of initial conditions of the process are rare, distant and deeply embedded in massive gas clumps, i.e., structures that eventually fragment into star clusters (Tan et al.

\footnotetext{
${ }^{1}$ Dept. of Astronomy, University of Florida, Gainesville, Florida 32611, USA

2 Depts. of Astronomy \& Physics, University of Florida, Gainesville, Florida 32611, USA

${ }^{3}$ Max-Planck-Institue for Extraterrestrial Physics, D-85748 Garching, Germany

${ }^{4}$ INAF - Osservatorio Astrosico di Arcetri, I-50125, Florence, Italy
} 
Conditions and Impact of Star Formation

2014). A key question is whether the formation mechanism is a scaled-up version of low-mass star formation. Core Accretion models assume it is: e.g., the Turbulent Core model (McKee \& Tan 2003 [MT03]) adopts an initial condition of a virialized massive starless core - a coherent gas cloud that will collapse via a central disk to form a single star or small- $N$ multiple. Alternatively, the Competitive Accretion model (Bonnell et al. 2001) forms massive stars at the center of a clump undergoing rapid global collapse and fragmentation mostly into a swarm of low-mass stars. A way to test the models is to identify and characterize initial conditions: do massive starless cores exist and, if so, what is their dynamical state?

However, a difficulty in such studies is estimating the strength of $B$-fields, which may provide significant support in addition to turbulence (note, thermal pressure is unimportant in these massive, cold clouds). While strong $B$-fields are seen around massive protostellar cores (e.g., Zhang et al. 2014), there are few measurements at earlier stages. Recently, Pillai et al. (2015) measured dynamically strong, $\sim 1 \mathrm{mG}$ $B$-fields in dark, presumably starless regions of Infrared Dark Clouds (IRDCs).

As a complementary approach, we attempt to measure core ages by astrochemical means, especially the deuteration fraction of key species, and compare these with dynamical (i.e., free-fall) timescales. If the chemical age is greater than the free-fall time, then we expect that a core is likely to have reached approximate virial equilibrium, so that any global contraction is at a relatively slow rate, perhaps regulated by $B$-field support. We also couple the chemical network to simple dynamical models to constrain the collapse rate relative to free-fall collapse.

The particular indicator we model is the deuterium fraction of $\mathrm{N}_{2} \mathrm{H}^{+}$, i.e., $D_{\text {frac }}^{\mathrm{N}_{2} \mathrm{H}^{+}} \equiv\left[\mathrm{N}_{2} \mathrm{D}^{+}\right] /\left[\mathrm{N}_{2} \mathrm{H}^{+}\right]$. It rises in cold, dense conditions of starless cores by $\gtrsim 4$ orders of magnitude above cosmic $[\mathrm{D}] /[\mathrm{H}] \sim 10^{-5}$. This is due to the parent exothermic reaction $\mathrm{p}-\mathrm{H}_{3}^{+}+\mathrm{HD} \rightleftharpoons \mathrm{p}-\mathrm{H}_{2} \mathrm{D}^{+}+\mathrm{p}-\mathrm{H}_{2}+232 \mathrm{~K}$ being favored at low

temperatures $(\lesssim 30 \mathrm{~K})$ (Pagani et al. 1992). $D_{\text {frac }}^{\mathrm{N}_{2} \mathrm{H}^{+}}$has been observed to be a good tracer of both low- and high-mass starless cores (e.g., Crapsi et al. 2005; Tan et al. 2013 [T13]), and is better than $D_{\text {frac }}^{\mathrm{HNC}}$ and $D_{\text {frac }}^{\mathrm{NH}_{3}}$ (Fontani et al. 2015). Other methods, such as dust continuum (e.g., Rathborne et al. 2006), dust extinction (e.g., Butler \& Tan 2012), are likely subject to contamination from the much more massive clump envelope surrounding the cores. Dust continuum emission is also more sensitive to warmer, protostellar cores, rather than starless cores.

\section{Results for the Massive Starless Cores C1-N and C1-S}

Two massive starless core candidates, $\mathrm{C} 1-\mathrm{N}$ and $\mathrm{C} 1-\mathrm{S}$, were identified in IRDC G028.37+00.07, hereafter IRDC C, from their $\mathrm{N}_{2} \mathrm{D}^{+}(3-2)$ emission observed with $A L M A$ by T13. Based on $1.3 \mathrm{~mm}$ dust continuum emission, C1-S is more massive, with $\sim 60 M_{\odot}$, while $\mathrm{C} 1-\mathrm{N}$ has $\sim 20 M_{\odot}$. The cores are $\sim 0.1 \mathrm{pc}$ in radius. C1-S is round and centrally concentrated (in both mm continuum and $\mathrm{N}_{2} \mathrm{D}^{+}(3-$ 2 ) integrated intensity), while C1-N appears more fragmented. Both cores are dark at wavelengths up to $100 \mu \mathrm{m}$ and there is no sign of star formation activity (e.g., from $\mathrm{SiO}(5-4)$ ). Velocity dispersions inferred from $\mathrm{N}_{2} \mathrm{D}^{+}(3-2)$ line widths 
are about half that of the fiducial MT03 Turbulent Core model, which assumes large-scale $B$-fields that imply an Alfvén Mach number of $m_{A}=1$. To achieve virial equilibrium would require stronger, $\sim 1 \mathrm{mG}$ fields, which imply $m_{A} \simeq 0.3$.

Follow-up observations of multiple transitions of $\mathrm{N}_{2} \mathrm{H}^{+}(1-0),(3-2),(4-3)$ and $\mathrm{N}_{2} \mathrm{D}^{+}(1-0)$ and (2-1) have been presented by Kong et al. (2015a [K15a]). Excitation temperatures of $\sim 4-7 \mathrm{~K}$ are derived for the $\mathrm{N}_{2} \mathrm{D}^{+}$line emission, resulting in a range of values of $D_{\text {frac }}^{\mathrm{N}_{2} \mathrm{H}^{+}} \simeq 0.15-0.72$ for C1-S and $\simeq 0.16-0.44$ for C1-N. However, additional factor of 2 uncertainties arise due to the difficulty in separating the $\mathrm{N}_{2} \mathrm{H}^{+}$emission of the cores from their more extended clump envelopes.

A gas-phase, spin-state astrochemical network was developed by Kong et al. (2015b [K15b]) to follow time evolution of $D_{\text {frac }}^{\mathrm{N}_{2} \mathrm{H}^{+}}$for given density $\left(n_{\mathrm{H}}\right)$, temperature $(T)$, cosmic ray ionization rate $(\zeta)$, extinction of Galactic FUV radiation $\left(A_{V}\right)$ and for either fixed or time-dependent heavy element gas phase depletion factor $\left(f_{D}\right)$ via freeze-out onto dust grain ice mantles. For fiducial conditions of $T \lesssim 15 \mathrm{~K}, \zeta=2.5 \times 10^{-17} \mathrm{~s}^{-1}, A_{V}=30 \mathrm{mag}$ and $f_{D}=10$ (i.e., gas phase underabundance by a factor of 10 ), the K15a results for chemical evolution at the densities of C1-S $\left(n_{\mathrm{H}} \simeq 6 \times 10^{5} \mathrm{~cm}^{-3}\right)$ and $\mathrm{C} 1-\mathrm{N}\left(n_{\mathrm{H}} \simeq 2 \times 10^{5} \mathrm{~cm}^{-3}\right)$ are that it takes $\gtrsim 1 \mathrm{Myr}$ to reach the equilibrium value of $D_{\text {frac,eq }}^{\mathrm{N}_{2} \mathrm{H}^{+}} \simeq 0.2-0.3$, if starting from an initial $\mathrm{OPR}^{\mathrm{H}_{2}}=3$. Starting from $\mathrm{OPR}^{\mathrm{H}_{2}}=0.01$ only leads to a factor of 2 reduction in this timescale. Note, the free-fall times for $\mathrm{C} 1-\mathrm{S}$ and $\mathrm{C} 1-\mathrm{N}$ are $(0.5$ and 1.0) $\times 10^{5} \mathrm{yr}$, respectively. Thus the cores are consistent with being in chemical equilibrium and this would have taken $\gtrsim 10$ local free-fall times to achieve.

K15a also present simple chemodynamical modeling of collapsing cores by controlling the density increase via $\mathrm{d} n_{\mathrm{H}}(t) / \mathrm{d} t=\alpha_{\mathrm{ff}} n_{\mathrm{H}}(t) / t_{\mathrm{ff}}(t)$, where $t_{\mathrm{ff}}$ is the local free-fall time at current density $n_{\mathrm{H}}$, and $\alpha_{\mathrm{ff}}$ is a dimensionless parameter setting the collapse rate relative to free-fall. These models are then also parameterized by a target final density, $n_{\mathrm{H}, 1}$, an initial density, $n_{\mathrm{H}, 0}$, an initial heavy element depletion factor, $f_{D, 0}$, and an initial $\mathrm{OPR}_{0}^{\mathrm{H}_{2}}$, with $T, \zeta$ and $A_{V}$ being held fixed.

K15b applied these chemodynamical models to the specific cases of C1-S and C1-N, with $T=10 \mathrm{~K}, A_{V}=30 \mathrm{mag}, \zeta=0.1$ to $10 \times 10^{-17} \mathrm{~s}^{-1}, \mathrm{OPR}_{0}^{\mathrm{H}_{2}}=0.01$ to $3, f_{D, 0}=1,3,10, n_{\mathrm{H}, 1}$ set equal to present-day observed densities, $n_{\mathrm{H}, 0}$ set to 10 or 100 times smaller, and $\alpha_{\mathrm{ff}}$ explored from 0.01 to 1 . Fig. 1 shows example results for $\alpha_{\mathrm{ff}}=0.01,0.033,0.1,0.33,1$ and $f_{D, 0}=1,3,10$ applied to C1-S in the parameter space of present-day $\left[\mathrm{N}_{2} \mathrm{D}^{+}\right], D_{\text {frac }}^{\mathrm{N}_{2} \mathrm{H}^{+}}$and $\left[\mathrm{o}-\mathrm{H}_{2} \mathrm{D}^{+}\right]$. The observed properties of C1-S, including an upper limit on $\left[\mathrm{o}-\mathrm{H}_{2} \mathrm{D}^{+}\right]$, also observed by K15a) are shown by red shaded regions. Models in or near the overlapped red areas are consistent with all constraints. $D_{\text {frac }}^{\mathrm{N}_{2} \mathrm{H}^{+}}$is the most stringent constraint and its high observed values of $\sim 0.3$ rules out fast collapsing $\alpha_{\mathrm{ff}}=1$ models, regardless of initial depletion factor. Models with $\alpha_{\mathrm{ff}} \lesssim 0.3$ give a much better match. In fact, the observations are broadly consistent with chemical equilibrium values, which the slow-collapsing models have time to converge to. From the broader parameter space exploration of K15b, it is concluded that the most likely evolutionary history of C1-N and C1-S involves collapse with $\alpha_{\mathrm{ff}} \lesssim 0.1$.

The above methods and results demonstrate the utility of using $\mathrm{N}_{2} \mathrm{D}^{+}$as a 


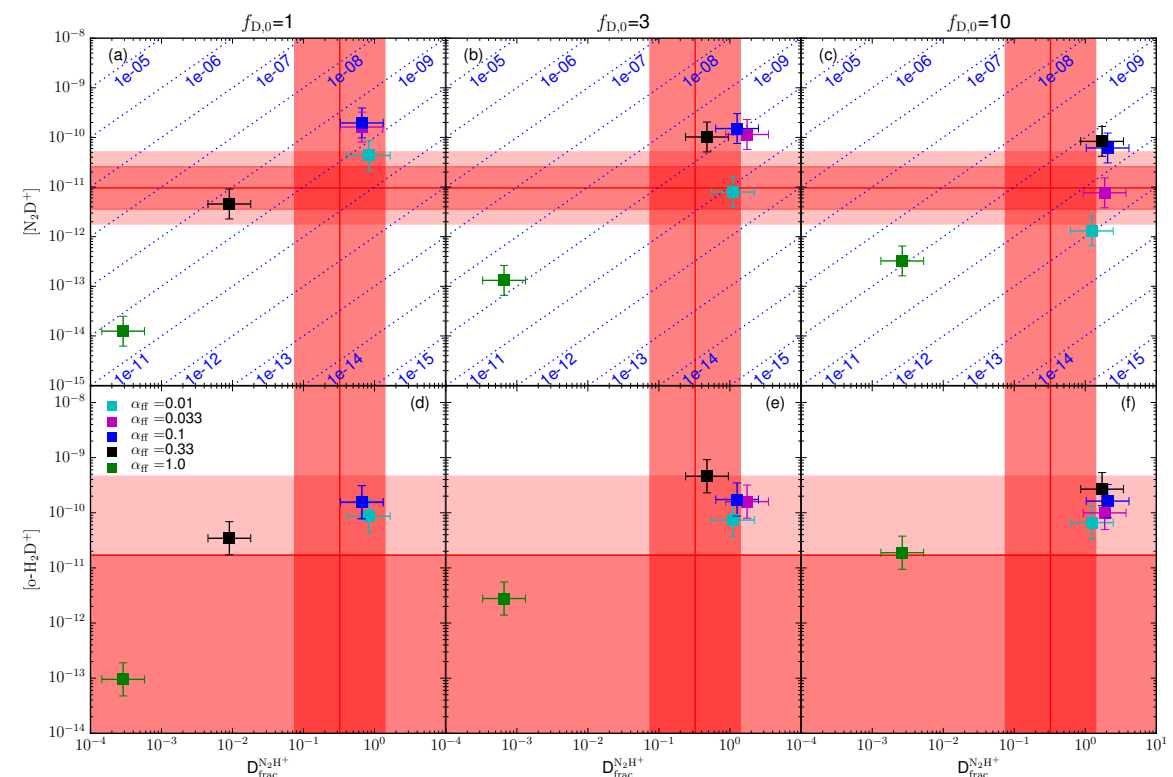

Fig. 1. (From K15b) C1-S fiducial models $\left(T=10 \mathrm{~K}, A_{V}=30 \mathrm{mag}, \zeta=2.5 \times\right.$ $10^{-17} \mathrm{~s}^{-1}, \mathrm{OPR}_{0}^{\mathrm{H}_{2}}=1, n_{\mathrm{H}, 0} / n_{\mathrm{H}, 1}=0.1$ with $f_{D, 0}=1,3,10$ [left, middle, right columns]) and observational constraints in the $\left[\mathrm{N}_{2} \mathrm{D}^{+}\right]-D_{\mathrm{frac}}^{\mathrm{N}_{2} \mathrm{H}^{+}}$(top row) and $\left[\mathrm{o}-\mathrm{H}_{2} \mathrm{D}^{+}\right]-D_{\text {frac }}^{\mathrm{N}_{2} \mathrm{H}^{+}}$ (bottom row) parameter space. The blue dotted lines in the top row show constant $\left[\mathrm{N}_{2} \mathrm{H}^{+}\right]$values. The red shaded areas show the observational constraints.

tracer of pre-stellar cores, especially in massive clump environments that exhibit extended $\mathrm{N}_{2} \mathrm{H}^{+}$emission. The ability to also estimate a deuteration age will also be helpful for assessing an unbiased pre-stellar core mass function, i.e., enabling accounting for the varying lifetimes of the cores.

\section{References}

Bonnell, I. A., Bate, M. R., Clarke, C. J., \& Pringle, J. E. 2001, MNRAS, 323, 785

Butler, M. J., \& Tan, J. C. 2012, ApJ, 754, 5

Crapsi, A., Caselli, P., Walmsley, M. C., et al. 2005, ApJ, 619, 379

Fontani, F., Busquet, G., Palau, A., et al. 2015, A\&A, 575, A87

Kong, S., Caselli, P., Tan, J. C., Wakelam, V., \& Sipilä, O. 2015b, ApJ, 804, 98 [K15b]

Kong, S., Tan, J. C., Caselli, P., et al. 2015a, arXiv:1509.08684 [K15a]

McKee, C. F., \& Tan, J. C. 2003, ApJ, 585, 850 [MT03]

Pagani, L., Salez, M., \& Wannier, P. G. 1992, A\&A, 258, 479

Pillai, T., Kauffmann, J., Tan, J. C., et al. 2015, ApJ, 799, 74

Rathborne, J. M., Jackson, J. M., \& Simon, R. 2006, ApJ, 641, 389

Tan, J. C., Beltrán, M. T., Caselli, P., et al. 2014, Protostars and Planets VI, 149

Tan, J. C., Kong, S., Butler, M. J., Caselli, P., \& Fontani, F. 2013, ApJ, 779, 96 [T13]

Zhang, Q., Qiu, K., Girart, J. M. et al. 2014, ApJ, 792, 116 